\title{
Challenges in Risk Assessment and Management of Veterinary Medicines for Plants: Gaps and Reflections for an Updated Approach
}

\author{
De la Torre $\mathrm{A}^{* 2}$, Haro $\mathbf{A}^{1}$, Carballo $\mathbf{M}^{2}$, de la Casa $\mathbf{I}^{1}$, Rubio $\mathrm{C}^{1}$, Cortés $\mathrm{G}^{1}$ and Carapeto $\mathrm{R}^{1}$ \\ ${ }^{1}$ Agencia Española de Medicamentos y Productos Sanitarios (AEMPS), Spain \\ ${ }^{2}$ INIA-CISA, Epidemiology and Environmental health, Spain
}

Received: May 31, 2018; Published: June 11, 2018

${ }^{*}$ Corresponding author: Ana de la Torre, INIA-CISA, Epidemiology and Environmental health, 28130, Valdeolmos, Spain

\section{Introduction}

Under the current legislation EPC [1], an Environmental Risk Assessment (ERA) is mandatory to obtain Marketing Authorization for Veterinary Medicinal Products (VMP). ERAs for VMP, plant protection products, biocides or industrial technical products, follow the General Protection Goals for ecosystems Devos [2]. It means that the ERA evaluates the environmental relevance of the active substances concentrations estimated in the different environmental compartments taking into account the ecotoxicological effects on representative organisms. Specifically, the guideline for VMP VICH GL 38, [3] mentions that "the overall target of the assessment is the protection of ecosystems". This General Protection Goal might be sufficient when no risks are identified, but when risks are identified a clearer definition of the protection goals is required in order to refine and manage such risks.

The most relevant scenario in the release assessment of VMP to the environment occurs in intensively farmed animal species. The manure produced by these animals is most frequently used across the EU as fertiliser in crops, although some countries may also use manure in grassland. However, during the exposure assessment, the definition of the different agro-livestock scenarios, the target plant species or the plant species that are more vulnerable and need a higher protection are not specific enough, which means that risk assessors have to interpret the General Protection Goals without clear guidance. In our view, the General Protection Goals need to be translated into Operational Protection Goals to achieve efficient and robust ERAs. This can be achieved focusing on the relevant agrolivestock scenarios. This paper aims to identify gaps of ERA of VMPs for terrestrial plants and offers proposals for applicable and suited Operational Protection Goals and Risk management measures.

\section{ERA of VMPs for Terrestrial Plants}

Considerable efforts were devoted in the past years to improve the ERA of VMPs for terrestrial plants resulting in a specific guideline where plant evaluation was refined EMA [4]. According to this, the effects of a VMP on plants are first assessed taking into account a deterministic approach (lower ecotoxicity end point from studies on 6 crop species belonging to different families).If a risk is found at this level the effects have to be refined in a higher tier by means of a probabilistic approach using the lower limit of the 5th percentile (LLHC5) of the ecotoxicity data distribution for 8 crop species. If the risk persists, then Risk Mitigation Measures (RMM) that reduces the exposure might be included in the Summary of Product Characteristics (SPC). If the RMM don't reduce the identified risk to an acceptable level, the benefits of the authorization must be weighed against the risks before an approval can be granted EPC [1]. Although the described procedure is a valuable tool, the risk for plants continues to be a critical point on the ERA of VMPs Kuster and Adler [5].

\section{Gaps identified in the Current Risk Assessment}

The efficient use of animal manure as soil fertilizer represents a good practice in agricultural and economic terms for sustainable agro-livestock production. The use of manure in the soil saves costs in chemical fertilizers and improves soil fertility and structure, contributing to the reduction of erosion problems, and enhancing crop yields. This is especially important in countries where the generation of organic by-products in farms is high, such as France, Germany, the United Kingdom and Spain Foged et al. [6], as it offers a viable option for their valuation. As stated above, the current Risk Assessment of VMPs considers that the source of emission of the active substances to the environment will be the use of manure as fertilizer in two different scenarios: Crops and grasslands.

The current Risk Assessment layout provides due protection to the grassland scenario. Grasslands are bio diverse scenarios that provide valuable ecosystem services as food web support, aesthetic values or genetic resources. The main endpoint is the 
maintenance of biodiversity Garcia-Alonso and Raybould [7] which in practical is achieved in the current Risk Assessment by means of the probabilistic approach that aims to protect $95 \%$ of vegetal species. In the crop scenario the identification of key plant species associated to agricultural uses of manure should be mandatory, as each crop field will based in a single plant species. Key plant species for a given scenario are major taxonomic or functional groups that support the landscape. The current Risk Assessment focuses in the protection of $95 \%$ of plant species. In our view, this constitutes a gap in the Risk Assessment of VMP as, in the crop scenario, the protection of crop plants, should be specially considered.

Manure management is mainly restricted to geographical areas where livestock activities are concentrated because manure transportation usually represents an additional cost. The most representative scenario for agricultural landscape in livestock areas is winter cereals according to the guideline for VMP VICH GL 38, [3], especially barley and wheat, although maize and sunflowers are becoming increasingly important Left et al. [8] In fact, around 50\% of the agricultural surface of the European Union is based in crop species of the Poaceae family (cereals and fodder) EUROSTAT [9]. This would constitute another gap in the Risk Assessment as the Poaceae family (monocotyledonous) might be underrepresented in the ecotoxicological data.

Off-crop areas (neighbouring crop areas with wild plant species where the manure is not intentionally applied), together with grasslands are especially relevant in the associated livestock activities, like apiculture. The maintenance of the flora biodiversity is a relevant parameter for the viability of honeybees and others pollinators in the Mediterranean scenario Asensio et al. [10]. This scenario is not specifically covered by the current Risk Assessment and this might be a third gap. Nevertheless it should be kept in mind that the protection of this specific scenario might be already considered when assessing the grassland scenario. According to the different entities of protection and spatial contexts, we can translate the General Protection Goal in two different Operational Protection Goals in order to cover the identified gaps. The first one, in crop-areas, will focus on the ecological functions that support the provisioning services of crop production. The second Operational Protection Goal, in the off-crop areas and grasslands, will focus in the maintenance of biodiversity to provide cultural, regulating and supporting services.

\section{Proposal of Operational}

Other regulatory frameworks, e.g. Plant Protection Products EC [11] have considered the human interests and the environmental interests when defining the protection goals for plants. To our view, it is useful for the risk assessment process bringing some aspects of this approach to the current ERA of VMPs. In the Risk Assessment of VMPs the General Protection Goal could also be translated into two Operational Protection Goals in order to cover the identified gaps:

Operational Protection Goal 1: The Protection of Human Interests: For the farmers' benefit, the first Operational Protection Goal should be ensuring that the VMP is not a risk for sustainable agricultural production. The current ERA aims to identify which plant families could be potentially more affected. If relevant, this information could be included in the Summary of Product Characteristics (e.g. "This VMP poses a risk for Cucurbitaceae and Alliaceae. The manure of treated animals should not be used to fertilize crops of these families").

Operational Protection Goal 2: The protection of Environmental Interests: As the current framework recommends the use of LLHC5 as the ecotoxicological endpoint of the probabilistic risk assessment to protect $95 \%$ of plant species, the protection of the structure and biodiversity of grassland is covered. Off-crop plants in the margins of the cropped area would also benefit from this protection goal.

Challenges for the implementation of Operational Protection Goals in plants: For the protection of sustainable agricultural production, the main challenges are

a) The identification of the key crop species over which manure is spread, and

b) To generate a risk-based approach based on the relevance of key crop species for production of food, feed and other products for human agricultural use. Regarding non-crop species, the interest of Protection Goals are grasslands and margins of cropped areas scenarios. The following issues should be addressed to improve the risk assessment and management for non-crop species:

i. Identification of appropriate assessment factor for the extrapolation of toxicity data from crop species to wild species or from the current endpoints to vegetative effects, and

ii. Exposure relevance to off-crop margins: considering the limited drift of the manure when applied to fields, the relevance of this scenario should be elucidated.

Methods to define the geographical significance of the proposed Operational Protection Goals are the first step towards a more substantial discussion. Hence, a good identification and interpretation of the agro-livestock scenarios across the EU are vital to deal with regional variations. Protection goals can be somehow abstract or global, what may turn them unfeasible when applied to very different agro geo climatic European zones. We propose a more practical and specific approach to set useful protection goals in the risk assessment for plants of veterinary medicines. It is possible to spatially identify and characterise the crop, grassland and offcrop scenarios considering the livestock density and distribution, the agricultural landscape and manure practices in each country. Standardised geo-referenced European database would give information about these driving factors and GIS-based modelling would allow defining the scenarios.

The production of these models would identify the representativeness of the main plant families exposed to VMP, the key plant species to be protected (target species) and the risk/ benefits of the different manure spreading methods. It would also allow the quantification of the impact on the environment 
according to the spatial differentiation of manure emissions and agro-livestock scenarios for policy formulation. It would be very valuable in order to refine risk: For instance, if the use of manure in grasslands is limited to specific countries/regions, a zonal risk assessment would be more informative than assuming the worsecase scenario, allowing determined regions to benefit from the use of manure as fertilizer.

\section{Tools for further Risk Assessment and Management}

In case that, during the assessment procedure, a risk is found for plants, the current scientific knowledge gives us additional tools for the refinement and management of such risk. It should also be taken into account that due to the persistence and mobility in soil and the continuous emission of active substances in manure, the accumulation in soil might also occur Zhang et al. [12]. This issue might somehow worsen the risk identified for plants. In a first step, available information on manure treatment methods could be employed for the management of the risk for plants. Composting is considered as one of the "best available techniques" for the onfarm processing of manure to avoid contamination associated to soil fertilization Foged et al. \& Giner et al. [6,13]. Its effectivity has been specifically demonstrated for some VMPs in avian manure Slana et al. [14]. Authors observed that when broiler manure is handled according to the usual manure management praxis, levels of antibiotic decrease and no risk for the terrestrial plants exists.

Another procedure would be to use spatially distributed models as an instrument to recommend not applying medicated manure on to hotspot areas where antibiotic accumulation can occur, increasing the risk for plants. It would depend on antibiotic emission (areas with high livestock density), soil characteristics, which vary across Europe, and antibiotic properties, which vary across drug classes. De la Torre et al. [15] developed soil maps for antibiotic contamination that show a high geographic variability. They can be successfully used for evaluating high and low risk areas within the EU and for designing interventions to mitigate the risk of VMPs. Risk models could also offer a great advantage in the preparation of monitoring protocols and surveillance programs. An example are the spatially implemented models for pesticides in soil, surface water and groundwater Tiktak et al. \& Pistocchi et al. $[16,17]$ which offer information on potential problematic areas for contamination. This is especially relevant for VMPs as current data relating to their occurrence, fate, transport and effects in the environment are poor and incomplete.

\section{Conclusion}

The proposed approach aims to improve the current ERA of VMPs for terrestrial plants considering the different agro-livestock scenarios in which manure is usually applied, and the target plant species to be protected by proposing refinement and mitigation tools. This, in turn, is expected to help decision-makers for more pragmatic judgements regarding differentiated land use and spatial risk mitigation measures.

\section{Acknowledgment}

This work has been funded by the Spanish Government (RTA2014-00012-C03-02), Community of Madrid (S2013/ABI2747 (TAVS-CM)) and Structural Funds of the European Union.

\section{References}

1. EPC (2001) European Parliament and the Council. Directive 2001/82/ EC on the Community code relating to veterinary medicinal products.

2. Devos Y (2015) Optimising environmental risk assessments. EMBO reports 16(9): 1060-1063.

3. VICH GL (2003) Guideline on environmental impact assessment for veterinary medicinal products phases II.

4. EMA (2011) European Medicines Agency. Concept paper on testing strategy and risk assessment for plants in the phase 2, of the environmental risk assessment for veterinary medical products.

5. Kuster A, Adler N (2014) Pharmaceuticals in the environment: scientific evidence of risks and its regulation. Phil Trans R Soc B 369: 20130587.

6. Foged Henning Lyngsø, Xavier Flotats, August Bonmati Blasi, Jordi Palatsi (2011) Inventory of manure processing activities in Europe. Technical Report No. I concern "Manure Processing Activities in Europe" to the European Commission. Directorate-General Environment p. 138.

7. Garcia Alonso M, Raybould A (2014) Protection goals in environmental risk assessment: a practical approach. Transgenic Res 23(6): 945-956.

8. Leff B, Ramankutty N, Foley JA (2004) Geographic distribution of major crops across the world. Global biogeochemical cycles 18(1): 21-27.

9. (2017) EUROSTAT (Statistical Offic of the European Union) Crop production in EU standard humidity.

10. Asensio I, Vicente Rubiano M, Muñoz MJ, Fernández-Carrión E, SánchezVizcaíno JM, et al. (2016) Importance of Ecological Factors and Colony Handling for Optimizing Health Status of Apiaries in Mediterranean Ecosystems. PloS one 11(10): e0164205.

11.EC (2002) European Commission Health \& Consumer Protection Directorate-General. Guidance Document on Terrestrial Ecotoxicology under Council Directive 91/414/EEC. SANCO/10329/2002 revs 2 final.

12. Zhang H, Li X, Yang Q, Sun L, Yang X, et al. (2017) Plant Growth, Antibiotic Uptake, and Prevalence of Antibiotic Resistance in an Endophytic System of Pakchoi under Antibiotic Exposure. Int J Environ Res Public Health 14(11): 1336

13. Giner G, Georgitzikis K, Scalet BM, Montobbio P, Roudier S, Delgado L (2017) Best Available Techniques (BAT) Reference Document for the Intensive Rearing of Poultry or Pigs. EUR 28674 EN.

14. Slana M, Žigon D, Sollner Dolenc M (2017) Enrofloxacin degradation in broiler chicken manure under field conditions and its residuals effects to the environment. Environ Sci Pollut Res 24(15): 13722-13731.

15. De la Torre A, Iglesias I, Carballo M, Ramírez P, Muñoz MJ (2012) An approach for mapping the vulnerability of European Union soils to antibiotic contamination. Science of the Total Environment 414: 672679.

16. Tiktak A, De Nie DS, Garcet JDP, Jones A, Vancloosterb M (2004) Assessment of pesticide leaching risk at the Pan-European level using a spatially disturbed model. The Europ PEARL approach. J Hidrology 289: 222-238.

17. Pistocchi A, Vizcaino P, Hauck M (2009) A GIS model-based screening of potential contamination of soil and water by pyrethroids in Europe. Journal of Environmental Management 90(11): 3410-3421. 
(c) (i) This work is licensed under Creative

Submission Link: https://biomedres.us/submit-manuscript.php

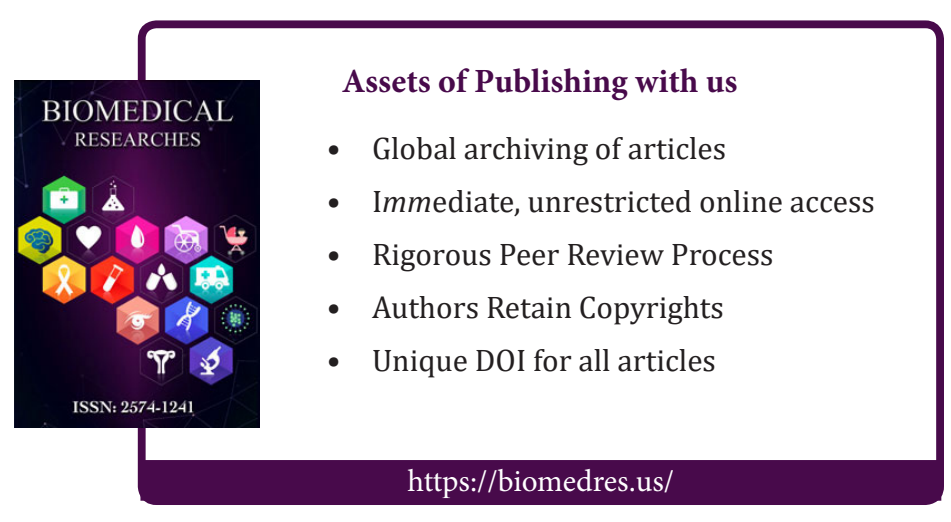

